\title{
Large linear negative thermal expansion in NiAs-type magnetic intermetallic Cr-Te-Se compounds
}

Jiawang $\mathrm{Xu}^{1}{ }^{1,}$, Xinqi Zheng ${ }^{1, \$}$, Shuxian Yang ${ }^{1,2}$, Lei Xi ${ }^{1}$, Shouguo Wang ${ }^{1, *}$, Lei Zhang ${ }^{3}$, Wenyun Yang, ${ }^{4, *}$, Jinbo Yang ${ }^{4}$, Xiaobai Ma ${ }^{5}$, Dongfeng Chen ${ }^{5}$, Lunhua He ${ }^{2,6,7}$, Sihao Deng ${ }^{7}$, Jingyan Zhang ${ }^{1}$, Yanfei $\mathrm{Wu}^{1}$, and Baogen Shen ${ }^{2,1,6}$

${ }^{1}$ Beijing Advanced Innovation Center for Materials Genome Engineering, School of Materials Science and Engineering, University of Science and Technology Beijing, Beijing 100083, China

${ }^{2}$ State key laboratory for Magnetism, Institute of Physics, Chinese Academy of Sciences \& University of Chinese Academy of Sciences, Beijing 100190, China

${ }^{3}$ Anhui Key Laboratory of Condensed Matter Physics at Extreme Conditions, High Magnetic Field Laboratory, Chinese Academy of Sciences, Hefei 230031, China

${ }^{4}$ State Key Laboratory for Mesoscopic Physics, School of Physics, Peking University, Beijing 100871, China

${ }^{5}$ Department of Nuclear Physics, China Institute of Atomic Energy, Beijing 102413, China

${ }^{6}$ Songshan Lake Materials Laboratory, Dongguan, Guangdong 523808, China

${ }^{7}$ Spallation Neutron Source Science Center, Dongguan, Guangdong 523803, China

Keywords: Magnetic transition, Negative thermal expansion, $\mathrm{CrTe}_{1-\mathrm{x}} \mathrm{Se}_{\mathrm{x}}$ compounds

\$) These authors contributed equally to this work.

${ }^{*}$ Author to whom correspondence should be addressed.

Electronic mail: sgwang@ustb.edu.cn, yangwenyun@pku.edu.cn 


\section{Supporting Information}

Table S1 Crystallographic Data (Powder): Lattice parameters, $\alpha=\beta=90^{\circ}, \quad \gamma=120^{\circ}$, space group : P63/mmc(194), atomic occupancy of $\mathrm{Cr}$, and refinement error for $\mathrm{CrTe}_{1-\mathrm{x}} \mathrm{Se}_{\mathrm{x}}(0 \leq \mathrm{x} \leq 0.15)$ obtained from XRD experiments with $\lambda=1.5406 \AA$ performed at $300 \mathrm{~K}$. Atomic positions: $\mathrm{Cr}: 2 \mathrm{a}(0,0,0)$; Te/Se: $2 \mathrm{c}(1 / 3,2 / 3,0.25)$.

\begin{tabular}{ccccc}
\hline \hline Samples & CrTe & $\mathrm{CrTe}_{\mathbf{0 . 5}} \mathbf{S e}_{\mathbf{0 . 0 5}}$ & $\mathbf{C r T e}_{\mathbf{0 . 9}} \mathbf{S e}_{\mathbf{0 . 1}}$ & $\mathbf{C r T e}_{\mathbf{0 . 8 5}} \mathbf{S e}_{\mathbf{0 . 1 5}}$ \\
\hline $\mathrm{a}(\AA)$ & $3.996(2)$ & $3.978(1)$ & $3.9614(9)$ & $3.9568(5)$ \\
$\mathrm{c}(\AA)$ & $6.233(3)$ & $6.225(2)$ & $6.218(2)$ & $6.2254(9)$ \\
$\mathrm{V}\left(\AA^{3}\right)$ & $86.20(0)$ & $85.33(6)$ & $84.50(5)$ & $84.41(2)$ \\
$\mathrm{Cr}$ occupancy & 0.86 & 0.86 & 0.85 & 0.88 \\
$\chi^{2}$ & 20.12 & 19.59 & 13.73 & 6.63 \\
wRp (\%) & 8.57 & 7.71 & 6.60 & 6.35 \\
$\mathrm{Rp}(\%)$ & 5.91 & 5.87 & 4.99 & 4.79 \\
radiation & $\mathrm{Cu}$ & $\mathrm{Cu}$ & $\mathrm{Cu}$ & $\mathrm{Cu}$ \\
\hline \hline
\end{tabular}


Table S2 Structural parameters of $\mathrm{CrTe}_{0.9} \mathrm{Se}_{0.1}$ at different temperatures based on NPD experiments. Space group P63/mmc. $\lambda=1.5406 \AA . \alpha=\beta=90^{\circ}, \gamma=120^{\circ}$, Atomic positions: Cr: 2a (0, 0, 0); Te/Se: 2c (1/3, 2/3, 0.25).

\begin{tabular}{|c|c|c|c|c|c|c|c|c|c|c|c|c|c|c|c|c|}
\hline Parameters & $150 \mathrm{~K}$ & $165 \mathrm{~K}$ & $180 \mathrm{~K}$ & $190 \mathrm{~K}$ & $195 \mathrm{~K}$ & $210 \mathrm{~K}$ & $225 \mathrm{~K}$ & $240 \mathrm{~K}$ & $255 \mathrm{~K}$ & $270 \mathrm{~K}$ & $285 K$ & $295 \mathrm{~K}$ & $300 \mathrm{~K}$ & $315 \mathrm{~K}$ & $330 \mathrm{~K}$ & $345 \mathrm{~K}$ \\
\hline$a(\AA)$ & $3.9734(2)$ & $3.9732(3)$ & $3.9725(3)$ & $3.9727(3)$ & $3.9730(3)$ & $3.9720(3)$ & $3.9711(3)$ & $3.9700(3)$ & $3.9692(3)$ & $3.9683(3)$ & $3.9680(3)$ & $3.9668(3)$ & $3.9668(3)$ & $3.9670(3)$ & $3.9672(3)$ & $3.9678(3)$ \\
\hline$c(\AA)$ & $6.2080(4)$ & $6.2104(5)$ & $6.2118(5)$ & $6.2140(5)$ & $6.2151(5)$ & $6.2164(5)$ & $6.2176(5)$ & $6.2195(5)$ & $6.2212(5)$ & $6.2227(5)$ & $6.2250(5)$ & $6.2256(5)$ & $6.2266(5)$ & $6.2281(5)$ & $6.2296(5)$ & $6.2315(5)$ \\
\hline$V\left(\AA^{3}\right)$ & $84.88(1)$ & $84.90(5)$ & $84.89(1)$ & $84.93(1)$ & $84.96(1)$ & $84.93(2)$ & $84.91(2)$ & $84.89(2)$ & $84.88(2)$ & $84.86(2)$ & $84.86(2)$ & $84.84(2)$ & $84.85(2)$ & $84.87(2)$ & $84.91(2)$ & $84.96(2)$ \\
\hline$C r_{-} M_{z}\left(\mu_{\mathrm{B}}\right)$ & $1.87(3)$ & $1.78(2)$ & $1.72(3)$ & $1.64(3)$ & $1.66(3)$ & $1.56(3)$ & $1.45(3)$ & $1.34(3)$ & $1.14(4)$ & $0.94(5)$ & $0.46(9)$ & $0.32(8)$ & 0 & 0 & 0 & 0 \\
\hline$w R p(\%)$ & 3.69 & 3.58 & 3.69 & 3.56 & 3.8 & 3.42 & 3.42 & 3.50 & 3.38 & 3.46 & 3.38 & 3.49 & 3.53 & 3.37 & 3.14 & 3.02 \\
\hline$R p(\%)$ & 2.72 & 2.65 & 2.73 & 2.64 & 2.79 & 2.46 & 2.48 & 2.55 & 2.45 & 2.46 & 2.45 & 2.25 & 2.55 & 2.47 & 2.44 & 2.34 \\
\hline$\chi^{2}$ & 4.308 & 4.128 & 4.343 & 4.078 & 5.190 & 4.242 & 4.351 & 4.411 & 4.171 & 4.346 & 4.218 & 4.474 & 4.543 & 4.119 & 3.435 & 3.184 \\
\hline
\end{tabular}



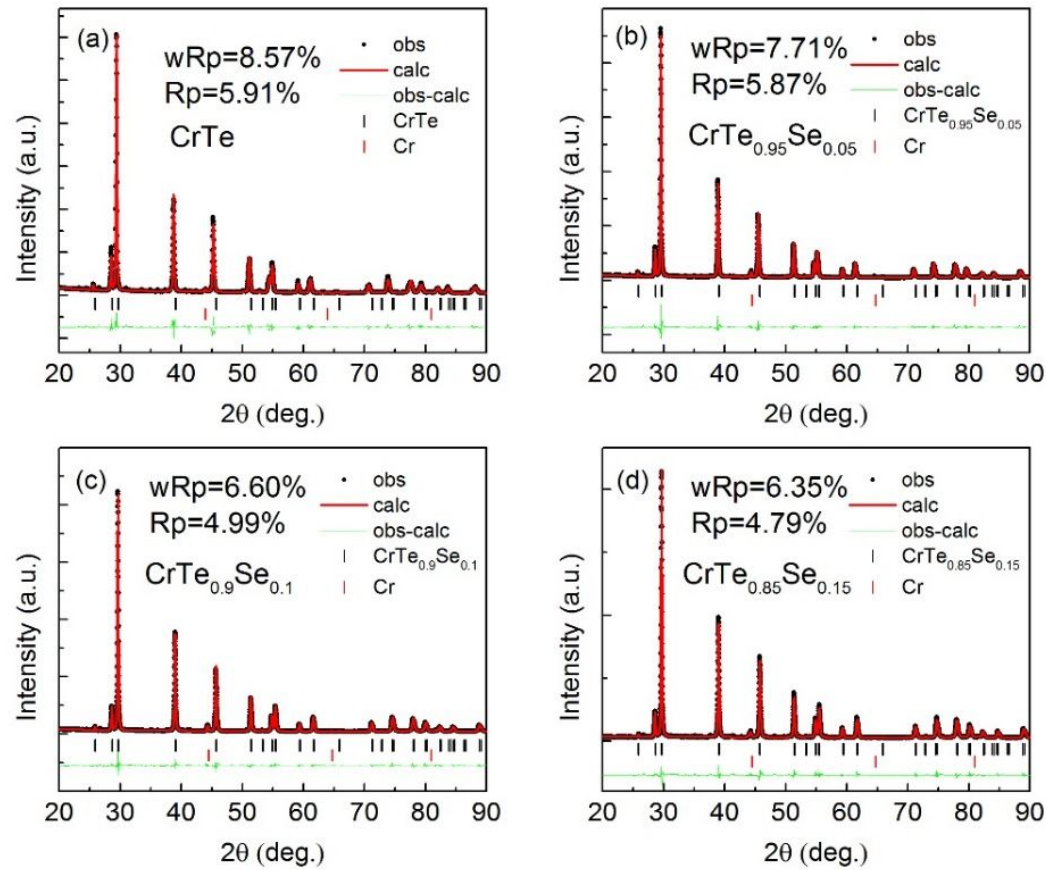

Figure S1 XRD patterns and fitting curves of $\mathrm{CrTe}_{1-\mathrm{x}} \mathrm{Se}_{\mathrm{x}}(0 \leq \mathrm{x} \leq 0.15)$ at $300 \mathrm{~K}$.

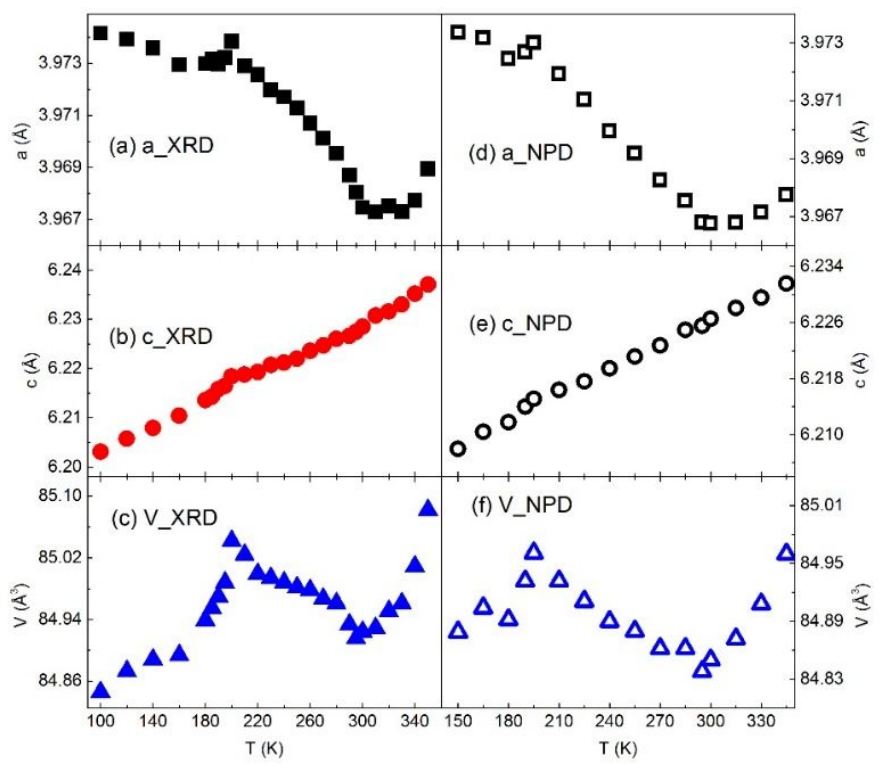

Figure S2 Temperature dependence of lattice parameters and unit cell volume for $\mathrm{CrTe}_{0.9} \mathrm{Se}_{0.1}$ obtained from XRD (a)-(c) and NPD (d)-(f) experiments. 

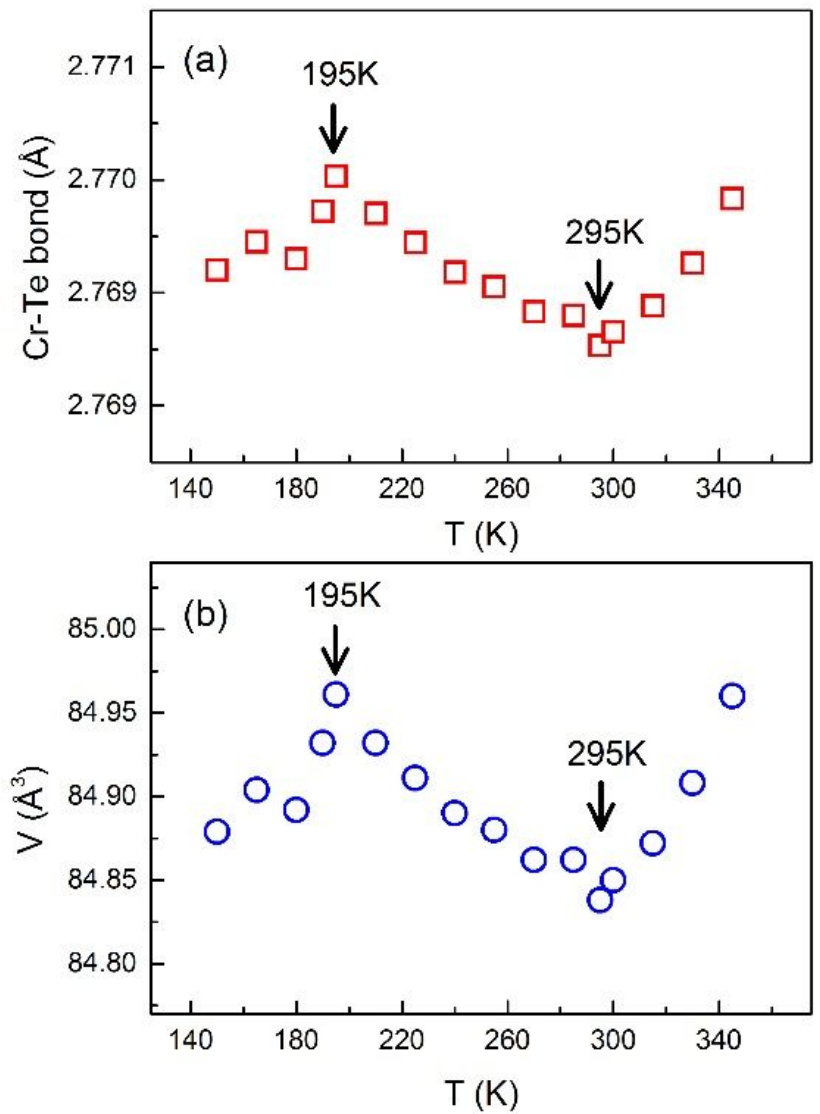

Figure S3 Temperature dependence of $\mathrm{Cr}$-Te bond and volume for $\mathrm{CrTe}_{0.9} \mathrm{Se}_{0.1}$ compound. 